\title{
THE MOSER-TRUDINGER INEQUALITY ON KÄHLER-EINSTEIN MANIFOLDS ${ }^{1}$
}

\author{
D.H. Phong \\ Department of Mathematics \\ Columbia University \\ New York, NY 10027 \\ Jacob Sturm \\ Department of Mathematics \\ Rutgers University \\ Newark, NJ 07102
}

\author{
Jian Song \\ Department of Mathematics \\ Johns Hopkins University \\ Baltimore, MD 21218 \\ Ben Weinkove ${ }^{2}$ \\ Department of Mathematics \\ Imperial College \\ London, SW7 2AZ, U.K.
}

\begin{abstract}
We prove the conjecture of Tian on the strong form of the Moser-Trudinger inequality for Kähler-Einstein manifolds with positive first Chern class, when there are no holomorphic vector fields, and, more generally, when the setting is invariant under a maximal compact subgroup of the automorphism group.
\end{abstract}

\section{Introduction}

The existence of canonical metrics in differential geometry is closely related to the properness of corresponding energy functionals. A basic example is given by Hermitian-Einstein metrics on holomorphic vector bundles. By the Donaldson-Uhlenbeck-Yau theorem [10], [26] existence is equivalent to the stability of the bundle. But existence is also equivalent to a certain non-linear bound from below for the Donaldson energy functional [11]. Another example is provided by Kähler-Einstein metrics. Such a metric always exists on a manifold $X$ with $c_{1}(X)<0$ [27], [1] and $c_{1}(X)=0$ [27]. If $c_{1}(X)>0$ then existence is conjectured by Yau to be equivalent to stability in the sense of geometric invariant theory [28]. Relating this to energy functionals, Tian showed in [22] that a Kähler-Einstein metric $\omega_{K E}$ exists in this case if and only if the functional $F_{\omega_{K E}}(\phi)$ is proper. More precisely, let $(X, \omega)$ be a compact Kähler manifold of dimension $n$ and volume $V$ with $c_{1}(X)>0$, and define the functionals $F_{\omega}(\phi)$ and $J_{\omega}(\phi)$ by

$$
\begin{aligned}
& F_{\omega}(\phi)=J_{\omega}(\phi)-\frac{1}{V} \int_{X} \phi \omega^{n}-\log \left(\frac{1}{V} \int_{X} e^{h_{\omega}-\phi} \omega^{n}\right), \\
& J_{\omega}(\phi)=\frac{\sqrt{-1}}{2 V} \sum_{j=0}^{n-1}\left(\frac{n-j}{n+1}\right) \int_{X} \partial \phi \wedge \bar{\partial} \phi \wedge \omega^{n-1-j} \wedge \omega_{\phi}^{j},
\end{aligned}
$$

\footnotetext{
${ }^{1}$ Research supported in part by National Science Foundation grants DMS-02-45371, DMS-05-14003 and DMS-05-04285.

${ }^{2}$ Currently on leave from Harvard University and supported by a Royal Society Research Assistantship.
} 
for all $\phi$ in $\mathcal{P}(X, \omega)=\left\{\phi \in C^{\infty}(X) \mid \omega_{\phi}=\omega+\frac{\sqrt{-1}}{2} \partial \bar{\partial} \phi>0\right\}$. Here $h_{\omega}$ is the potential for the Ricci curvature of the Kähler form $\omega$ defined by

$$
\operatorname{Ric}(\omega)-\omega=\frac{\sqrt{-1}}{2} \partial \bar{\partial} h_{\omega}, \quad \int_{X} e^{h_{\omega}} \omega^{n}=V .
$$

Then it is shown in [22] that, under the assumption that $X$ admits no non-trivial holomorphic vector fields, the existence of a Kähler-Einstein metric $\omega_{K E}$ is equivalent to the existence of a Kähler metric $\omega \in c_{1}(X)$, a strictly positive exponent $\gamma$, and corresponding constants $A_{\gamma}>0, B_{\gamma}$, so that

$$
F_{\omega}(\phi) \geq A_{\gamma} J_{\omega}(\phi)^{\gamma}-B_{\gamma}
$$

for all $\phi$ in $\mathcal{P}(X, \omega)$. In view of the cocycle identity, $F_{\omega}\left(\phi_{1}+\phi_{2}\right)=F_{\omega}\left(\phi_{1}\right)+F_{\omega_{\phi_{1}}}\left(\phi_{2}\right)$ and properties of the functional $J_{\omega}$ (see section 2), the inequality (1.3) holds for every $\omega \in c_{1}(X)$ if and only if it holds for some $\omega \in c_{1}(X)$.

Inequalities such as (1.3) are sometimes referred to as generalized Moser-Trudinger inequalities, by analogy with similar identities on the sphere $S^{2}$. Indeed, the Moser-Trudinger inequality [15], [25], [16] for functions $u \in L_{1}^{2}\left(S^{2}\right)$,

$$
\frac{1}{V} \int_{S^{2}} e^{-u} \omega_{K E} \leq C e^{\frac{\nu}{4 V} \int_{S^{2}} \sqrt{-1} \partial u \wedge \bar{\partial} u-\frac{1}{V} \int_{S^{2}} u \omega_{K E}}
$$

with the optimal constants $C=\nu=1$ and $\omega_{K E}$ the round metric on $S^{2}$, implies $F_{\omega_{K E}}(\phi) \geq$ 0 for $\phi \in \mathcal{P}\left(S^{2}, \omega_{K E}\right)$. Aubin [2] showed that if $u$ satisfies

$$
\int_{S^{2}} \lambda e^{-u} \omega_{K E}=0, \quad \text { for all } \lambda \in \Lambda,
$$

where $\Lambda$ is the space of eigenfunctions of the Laplacian $\Delta_{\omega_{K E}}$ with eigenvalue 1 , then the constant $\nu$ can be reduced to $1 / 2+\epsilon$ for any $\epsilon>0$, at the expense of increasing $C$. See also [7] for further results along these lines. The inequality (1.3) for $\gamma=1$ and $\omega=\omega_{K E}$ corresponds to a reduction in the constant $\nu$ to $\nu=1-A_{1}$, with $C=e^{B_{1}}$.

The exponent $\gamma$ found for (1.3) in [22] was $\gamma=e^{-n} /\left(8 n+8+e^{-n}\right)$. In [6] it has been improved to $\gamma=1 /(4 n+5)$. It was conjectured in [22] that the optimal exponent should be $\gamma=1$. It has been shown in [19] that this is indeed the case when the $\alpha$-invariant [21], [23] is strictly greater than $\frac{n}{n+1}$. Also, very recently, it has been shown [29] that the conjecture holds in the case of toric manifolds for potentials invariant under the compact torus action.

In this paper, we prove Tian's conjecture in general.

Theorem 1 Assume $c_{1}(X)>0, X$ has no non-trivial holomorphic vector fields and that $X$ admits a Kähler-Einstein metric $\omega_{K E}$. Then there exist positive constants $A$ and $B$ such that

$$
F_{\omega_{K E}}(\phi) \geq A J_{\omega_{K E}}(\phi)-B
$$

for all $\phi \in \mathcal{P}\left(X, \omega_{K E}\right)$. 
If $X$ does have non-trivial holomorphic vector fields, Theorem 1 can be generalized as follows (cf. [22], Theorem 4.4). Let $\operatorname{Aut}^{0}(X)$ be the component of the automorphism group of $X$ containing the identity, and $G=\operatorname{Stab}\left(\omega_{K E}\right) \subset \operatorname{Aut}^{0}(X)$ be the stabilizer of $\omega_{K E}$.

Theorem 2 Assume $c_{1}(X)>0$ and that $X$ admits a Kähler-Einstein metric $\omega_{K E}$. Assume that $\operatorname{Aut}^{0}(X)$ has a finite center. Then there exist positive constants $A$ and $B$ such that

$$
F_{\omega_{K E}}(\phi) \geq A J_{\omega_{K E}}(\phi)-B
$$

for all $G$-invariant potentials $\phi \in \mathcal{P}\left(X, \omega_{K E}\right)$. More generally, if $G \subset \operatorname{Stab}\left(\omega_{K E}\right)$ is a closed subgroup whose centralizer in $\operatorname{Stab}\left(\omega_{K E}\right)$ is finite, then the same inequality (1.7) holds for all $G$-invariant potentials $\phi$.

By the results of [12], [14] and [4], $\operatorname{Stab}\left(\omega_{K E}\right)$ is a maximal compact subgroup of $\operatorname{Aut}^{0}(X)$ and the complexification of its Lie algebra is the space of holomorphic vector fields.

The proof of Theorem 1 consists of two steps:

Step 1. Prove the inequality (1.3) for some $\gamma>0$ and for those $\phi$ satisfying

$$
\operatorname{Osc}(\phi) \leq K\left(J_{\omega_{K E}}(\phi)+1\right)
$$

for some constant $K$, where the $A_{\gamma}$ and $B_{\gamma}$ now depend on $K$.

Step 2. Remove the assumption (1.8) and prove the Moser-Trudinger inequality (1.3) with $\gamma=1$.

- Tian [22] has given a proof of the first step with $\gamma=e^{-n} /\left(8 n+8+e^{-n}\right)$. His proof makes use of the reverse continuity method of Bando-Mabuchi [4] and a modification of Bando's smoothing by the Kähler-Ricci flow [3]. We have taken the opportunity to present a complete and somewhat more streamlined proof of Step 1 in sections 2-4. There are some simplifications and improvements, and in particular we have eliminated the two Moser iteration arguments used in [22]. As a consequence, $\gamma$ here can be taken to be arbitrarily close to $1 /(2 n-1)$ if $n>1$ and arbitrarily close to $\frac{1}{2}$ if $n=1$, an improvement over previously known results.

- It was shown by Tian-Zhu [24] that the assumption (1.8) can be removed so that (1.3) holds with the $\gamma$ of Step 1. In section 5 we prove Step 2 by simultaneously removing the assumption and proving the full Moser-Trudinger inequality with $\gamma=1$, regardless of the value of $\gamma$ obtained in Step 1. A reader who is familiar with the afore-mentioned result of [22] and wants to immediately see a proof of Theorem 1 may wish to skip to this section.

- Finally we show, also in section 5 , how to prove Theorem 2 by making some minor modifications to the proof of Theorem 1.

It may be interesting to have a version of Theorem 2 with the assumption of G-invariance replaced by a less restrictive orthogonality condition on $\phi$. If one considers the condition $\phi \perp \Lambda$, then one would have to show that the projection of $\psi_{t}$ on $\Lambda$ is controlled by the 
projection of $\psi_{t}$ on $\Lambda^{\perp}$, where $\psi_{t}$ are the potentials introduced in section 3 below. Note that the condition $\phi \perp \Lambda$ is different from the condition $e^{-\phi} \perp \Lambda$ introduced in [2]. It is known that (1.3) does not hold (for any exponent $\gamma>0$ ) if the plurisubharmonicity condition $\omega_{K E}+\frac{\sqrt{-1}}{2} \partial \bar{\partial} \phi>0$ is dropped, as there are counterexamples [17] with $\phi \perp \Lambda$. Other non-plurisubharmonic counterexamples with $\phi \perp \Lambda$ can also be deduced from the results of $[5]$.

Remark The results above also immediately imply analogous inequalities for the Mabuchi energy [13] by the argument in [22] and the $E_{1}$ functional [8] by the argument in [20].

\section{Proof of Step 1}

We assume the existence of a Kähler-Einstein metric $\omega_{K E}$, and establish the inequality (1.3) for $F_{\omega_{K E}}(\phi)$, for $\phi$ satisfying (1.8). Fix a potential $\phi \in \mathcal{P}\left(X, \omega_{K E}\right)$, and set $\omega=$ $\omega_{K E}+\frac{\sqrt{-1}}{2} \partial \bar{\partial} \phi$. Consider the following complex Monge-Ampère equation,

$$
\left(\omega+\frac{\sqrt{-1}}{2} \partial \bar{\partial} \phi_{t}\right)^{n}=e^{h_{\omega}-t \phi_{t}} \omega^{n}, \quad t \in[0,1]
$$

By a theorem of Bando-Mabuchi [4], since there are no non-trivial holomorphic vector fields, a unique solution $\phi_{t}$ to this equation exists for all $t \in[0,1]$, and $\omega_{\phi_{1}}=\omega_{K E}$. In particular $\phi_{1}$ and $-\phi$ differ by a constant. To exploit this equation, we require another functional $I_{\omega}(\phi)$, defined by

$$
I_{\omega}(\phi)=\frac{\sqrt{-1}}{2 V} \sum_{j=0}^{n-1} \int_{X} \partial \phi \wedge \bar{\partial} \phi \wedge \omega^{j} \wedge \omega_{\phi}^{n-1-j}=\frac{1}{V} \int_{X} \phi\left(\omega^{n}-\omega_{\phi}^{n}\right) .
$$

From the explicit expressions for $I_{\omega}$ and $J_{\omega}$, it is easy to see that $I_{\omega}, J_{\omega}$ are always positive, and that

$$
\frac{1}{n} J_{\omega} \leq \frac{1}{n+1} I_{\omega} \leq J_{\omega}
$$

The derivatives of these functionals $I_{\omega}, J_{\omega}$ along a general path $\phi_{t}$ are given by

$$
\begin{aligned}
\frac{d}{d t} J_{\omega}\left(\phi_{t}\right) & =\frac{1}{V} \int_{X} \dot{\phi}_{t}\left(\omega^{n}-\omega_{\phi_{t}}^{n}\right), \\
\frac{d}{d t} I_{\omega}\left(\phi_{t}\right) & =\frac{1}{V} \int_{X} \dot{\phi}_{t}\left(\omega^{n}-\omega_{\phi_{t}}^{n}\right)-\frac{1}{V} \int_{X} \phi_{t} \frac{d}{d t}\left(\omega_{\phi_{t}}^{n}\right) .
\end{aligned}
$$

As a first consequence of these formulas, we find

$$
\frac{d}{d t}\left(I_{\omega}-J_{\omega}\right)\left(\phi_{t}\right)=-\frac{d}{d t}\left(\frac{1}{V} \int_{X} \phi_{t} \omega_{\phi_{t}}^{n}\right)+\frac{1}{V} \int_{X} \dot{\phi}_{t} \omega_{\phi_{t}}^{n}
$$


When $\phi_{t}$ satisfies the Monge-Ampère equation (2.1), we have $\int_{X}\left(t \dot{\phi}_{t}+\phi_{t}\right) \omega_{\phi_{t}}^{n}=0$, which follows from differentiating the equation $\int_{X} e^{h_{\omega}-t \phi_{t}} \omega^{n}=V$. Substituting for $\int_{X} \dot{\phi}_{t} \omega_{\phi_{t}}^{n}$ in (2.6) and integrating in $t$, we obtain [9] for all $t$

$$
-\frac{1}{V} \int_{X} \phi_{t} \omega_{\phi_{t}}^{n}=\left(I_{\omega}-J_{\omega}\right)\left(\phi_{t}\right)-\frac{1}{t} \int_{0}^{t}\left(I_{\omega}-J_{\omega}\right)\left(\phi_{s}\right) d s
$$

or equivalently, using the expression (2.2) for $I_{\omega}\left(\phi_{t}\right)$,

$$
F_{\omega}\left(\phi_{t}\right)=-\frac{1}{t} \int_{0}^{t}\left(I_{\omega}-J_{\omega}\right)\left(\phi_{s}\right) d s-\log \left(\frac{1}{V} \int_{X} e^{h_{\omega}-\phi_{t}} \omega^{n}\right) .
$$

Taking $t=1$, the last term on the right hand side is zero, and $F_{\omega}\left(\phi_{1}\right)=-F_{\omega_{K E}}(\phi)$, so that

$$
F_{\omega_{K E}}(\phi)=\int_{0}^{1}\left(I_{\omega}-J_{\omega}\right)\left(\phi_{t}\right) d t
$$

Next, the variational formulas for $I_{\omega}$ and $J_{\omega}$ also imply

$$
\frac{d}{d t}\left(I_{\omega}-J_{\omega}\right)\left(\phi_{t}\right)=-\frac{1}{V} \int_{X} \phi_{t} \frac{d}{d t}\left(\omega_{\phi_{t}}^{n}\right)=-\frac{1}{V} \int_{X} \phi_{t}\left(\Delta_{t} \dot{\phi}_{t}\right) \omega_{\phi_{t}}^{n},
$$

where $\Delta_{t}$ is the scalar Laplacian with respect to the Kähler form $\omega_{\phi_{t}}$. Differentiating the Monge-Ampère equation (2.1) shows that $\Delta_{t} \dot{\phi}_{t}=-t \dot{\phi}_{t}-\phi_{t}$, and hence

$$
\frac{d}{d t}\left(I_{\omega}-J_{\omega}\right)\left(\phi_{t}\right)=\frac{1}{V} \int_{X}\left(-\Delta_{t} \dot{\phi}_{t}\right)\left(-\Delta_{t}-t\right) \dot{\phi}_{t} \omega_{\phi_{t}}^{n}
$$

But the Monge-Ampère equation (2.1) also implies that $\operatorname{Ric}\left(\omega_{\phi_{t}}\right)=t \omega_{\phi_{t}}+(1-t) \omega \geq t \omega_{\phi_{t}}$, and hence, by the Bochner-Kodaira formula, $-\Delta_{t}-t$ is a positive operator. It follows that the right hand side of $(2.11)$ is nonnegative and $\left(I_{\omega}-J_{\omega}\right)\left(\phi_{t}\right)$ is a nondecreasing function of $t$.

- We will also need the following consequence of the fact that the functional $F_{\omega}^{0}$ defined by $F_{\omega}^{0}(\phi)=J_{\omega}(\phi)-\frac{1}{V} \int_{X} \phi \omega^{n}$ also satisfies the cocycle property $F_{\omega}^{0}(\psi+\theta)=F_{\omega}^{0}(\psi)+F_{\omega_{\psi}}^{0}(\theta)$ :

$$
\left|J_{\omega}\left(\phi_{1}\right)-J_{\omega}\left(\phi_{0}\right)\right| \leq \operatorname{osc}\left(\phi_{1}-\phi_{0}\right), \quad \text { for } \phi_{0}, \phi_{1} \in \mathcal{P}(X, \omega) .
$$

Indeed,

$$
\begin{aligned}
J_{\omega}\left(\phi_{1}\right)-J_{\omega}\left(\phi_{0}\right) & =\frac{1}{V} \int_{X}\left(\phi_{1}-\phi_{0}\right) \omega^{n}-F_{\omega_{\phi_{1}}}^{0}\left(\phi_{0}-\phi_{1}\right) \\
& \leq \frac{1}{V} \int_{X}\left(\phi_{1}-\phi_{0}\right) \omega^{n}+\frac{1}{V} \int_{X}\left(\phi_{0}-\phi_{1}\right) \omega_{\phi_{1}}^{n} \leq \operatorname{osc}\left(\phi_{1}-\phi_{0}\right),
\end{aligned}
$$

where we made use in the inequality of the fact that $J \geq 0$. Interchanging $\phi_{0}$ and $\phi_{1}$ establishes the desired estimate. There is a similar inequality for the functional $\left(I_{\omega}-J_{\omega}\right)$ which will also be useful later:

$$
\left|\left(I_{\omega}-J_{\omega}\right)\left(\phi_{1}\right)-\left(I_{\omega}-J_{\omega}\right)\left(\phi_{0}\right)\right| \leq n \operatorname{osc}\left(\phi_{1}-\phi_{0}\right) .
$$


To see this, note that adding

$$
I_{\omega}\left(\phi_{0}\right)-I_{\omega}\left(\phi_{1}\right)=\frac{1}{V} \int_{X}\left(\phi_{0}-\phi_{1}\right) \omega^{n}+\frac{1}{V} \int_{X}\left(\phi_{1}-\phi_{0}\right) \omega_{\phi_{1}}^{n}+\frac{1}{V} \int_{X} \phi_{0}\left(\omega_{\phi_{1}}^{n}-\omega_{\phi_{0}}^{n}\right),
$$

to $(2.13)$ gives

$$
\begin{aligned}
\left(I_{\omega}-J_{\omega}\right)\left(\phi_{0}\right)-\left(I_{\omega}-J_{\omega}\right)\left(\phi_{1}\right) & \leq \frac{1}{V} \int_{X} \phi_{0}\left(\omega_{\phi_{1}}^{n}-\omega_{\phi_{0}}^{n}\right) \\
& =\frac{1}{V} \int_{X}\left(\phi_{1}-\phi_{0}\right)\left(\omega_{\phi_{0}}-\omega\right) \wedge \sum_{j=0}^{n-1} \omega_{\phi_{0}}^{j} \wedge \omega_{\phi_{1}}^{n-1-j},
\end{aligned}
$$

after integrating by parts, and this is bounded above by $n \operatorname{osc}\left(\phi_{1}-\phi_{0}\right)$. Interchanging $\phi_{1}$ and $\phi_{0}$ gives $(2.14)$.

We return now to the proof of the inequality (1.3). Since $\left(I_{\omega}-J_{\omega}\right)\left(\phi_{t}\right)$ is nondecreasing in $t$, the identity (2.9) implies that, for any $t$ in $[0,1]$,

$$
F_{\omega_{K E}}(\phi) \geq(1-t)(I-J)_{\omega}\left(\phi_{t}\right) \geq \frac{1}{n}(1-t) J_{\omega}\left(\phi_{t}\right)
$$

where we have made use of (2.3). Since $\left|J_{\omega}\left(\phi_{t}\right)-J_{\omega}\left(\phi_{1}\right)\right| \leq \operatorname{osc}\left(\phi_{t}-\phi_{1}\right)$ and $J_{\omega}\left(\phi_{1}\right)=$ $J_{\omega_{K E}}(\phi)$, we deduce that

$$
F_{\omega_{K E}}(\phi) \geq \frac{1}{n}(1-t) J_{\omega_{K E}}(\phi)-\frac{1}{n}(1-t) \operatorname{osc}\left(\phi_{t}-\phi_{1}\right)
$$

- The main problem is then to estimate $\operatorname{osc}\left(\phi_{t}-\phi_{1}\right)$. It is of course sufficient to estimate $\left\|\phi_{1}-\phi_{t}\right\|_{C^{0}}$. The original Monge-Ampère equation (2.1) is written in terms of $\omega_{\phi_{t}} \equiv$ $\omega+\frac{\sqrt{-1}}{2} \partial \bar{\partial} \phi_{t}$, so that $\omega$ is the reference metric. Since the key estimates which we need take place near $t=1$, and since $\omega$ depends on the given potential $\phi \in \mathcal{P}\left(X, \omega_{K E}\right)$, it is preferable to rewrite (2.1) as a Monge-Ampère equation with $\omega_{K E}$ as reference metric. It is easily verified using (2.1) that

$$
\log \frac{\omega_{K E}^{n}}{\left(\omega_{K E}-\frac{\sqrt{-1}}{2} \partial \bar{\partial}\left(\phi_{1}-\phi_{t}\right)\right)^{n}}+\left(\phi_{1}-\phi_{t}\right)=(t-1) \phi_{t},
$$

For later use, we also compare $(t-1) \phi_{t}$ with the potential function $h_{\omega_{\phi_{t}}}$ for the Ricci curvature with the normalization (1.2). We must have $h_{\omega_{\phi_{t}}}=(t-1) \phi_{t}+c_{t}$ for some constant $c_{t}$. The normalization condition (1.2) gives

$$
\int_{X} \omega_{\phi_{t}}^{n}=\int_{X} e^{h_{\omega_{\phi_{t}}}} \omega_{\phi_{t}}^{n}=\int_{X} e^{(t-1) \phi_{t}+c_{t}} \omega_{\phi_{t}}^{n}
$$

from which it follows that $\left|c_{t}\right| \leq(1-t)\left\|\phi_{t}\right\|_{C^{0}}$. In particular,

$$
\left\|h_{\omega_{\phi_{t}}}\right\|_{C^{0}} \leq 2(1-t)\left\|\phi_{t}\right\|_{C^{0}}
$$


- The equation (2.18) is of the form,

$$
\log \frac{\omega_{K E}^{n}}{\left(\omega_{K E}-\frac{\sqrt{-1}}{2} \partial \bar{\partial} \psi\right)^{n}}+\psi=h,
$$

with $\psi=\phi_{1}-\phi_{t}$ and $h=(t-1) \phi_{t}$. As a first attempt to get estimates for $\left\|\phi_{1}-\phi_{t}\right\|_{C^{0}}$ in terms of $1-t$ and $\left\|\phi_{t}\right\|_{C^{0}}$, we can apply the implicit function theorem. The linearization of the left hand side of $(2.21)$ at $\psi=0$ is $\delta \psi \mapsto \Delta_{K E} \delta \psi+\delta \psi$, which is an invertible operator from $L_{k+2}^{p}(X) \rightarrow L_{k}^{p}(X)$ and from $C^{2, \kappa}(X) \rightarrow C^{\kappa}(X)$, for any $p>1, k \geq 0$ and $0<\kappa<1$, when $X$ has no non-trivial holomorphic vector fields. Thus there exist constants $C\left(\omega_{K E}\right)$, $\epsilon\left(\omega_{K E}\right)>0$ (depending on $p, k$, and $\kappa$ ) so that if $\psi$ and $h$ satisfy the equation (2.21), then

$$
\|h\|_{C^{0, \kappa}} \leq \epsilon\left(\omega_{K E}\right) \Rightarrow\|\psi\|_{C^{2, \kappa}} \leq C\left(\omega_{K E}\right)\|h\|_{C^{0, \kappa}}
$$

and

$$
\|h\|_{L_{k}^{p}} \leq \epsilon\left(\omega_{K E}\right) \Rightarrow\|\psi\|_{L_{k+2}^{p}} \leq C\left(\omega_{K E}\right)\|h\|_{L_{k}^{p}}
$$

The above norms, and indeed any other unadorned norms appearing in this paper, are understood to be taken with respect to the metric $\omega_{K E}$. Now for $p>2 n$ and $k=0$, we obtain, in view of the Sobolev imbedding theorem,

$$
\|h\|_{C^{0}} \leq \epsilon\left(\omega_{K E}\right) \Rightarrow\|\psi\|_{C^{0}} \leq C\left(\omega_{K E}\right)\|h\|_{C^{0}}
$$

Applied to the equation (2.18), we find

$$
(1-t)\left\|\phi_{t}\right\|_{C^{0}} \leq \epsilon\left(\omega_{K E}\right) \Rightarrow\left\|\phi_{1}-\phi_{t}\right\|_{C^{0}} \leq C\left(\omega_{K E}\right)(1-t)\left\|\phi_{t}\right\|_{C^{0}} .
$$

If indeed $(1-t)\left\|\phi_{t}\right\|_{C^{0}} \leq \epsilon\left(\omega_{K E}\right)$ for all $t$, then we pick say $t=\frac{1}{2}$ in the inequality (2.17), and the desired inequality (1.6) follows at once. Otherwise, we let $t_{0}$ be the largest value possible so that $(1-t)\left\|\phi_{t}\right\|_{C^{0}}<\epsilon\left(\omega_{K E}\right)$ for all $t \in\left(t_{0}, 1\right]$. Clearly $t_{0}<1$, although it does depend on the potential $\phi \in \mathcal{P}\left(X, \omega_{K E}\right)$. Assuming without loss of generality that both $\epsilon\left(\omega_{K E}\right)$ and $1-t_{0}$ are small numbers, we can deduce that $\frac{1}{2}\left\|\phi_{t_{0}}\right\|_{C^{0}} \leq\left\|\phi_{1}\right\|_{C^{0}} \leq 2\left\|\phi_{t_{0}}\right\|_{C^{0}}$, and hence

$$
F_{\omega_{K E}}(\phi) \geq \frac{\epsilon\left(\omega_{K E}\right)}{2 n} \frac{J_{\omega_{K E}}(\phi)}{\left\|\phi_{1}\right\|_{C^{0}}}-C^{\prime}
$$

However, in general, $\left\|\phi_{1}\right\|_{C^{0}}$ is of the same size as $J_{\omega_{K E}}$, so this estimate does not improve over the already known fact that $F_{\omega_{K E}}(\phi)$ is bounded from below.

- The idea is to improve on the range of $t$ on the left side of the implication in (2.25) so that, roughly speaking, $(1-t) \sim 1 /\left\|\phi_{t}\right\|_{C^{0}}^{1-\gamma}$ for some $\gamma>0$, assuming $\left\|\phi_{t}\right\|_{C^{0}}$ is large. More precisely, we have the following lemma, which provides the key estimate for $\left\|\phi_{1}-\phi_{t}\right\|_{C^{0}}$ :

Lemma 1 Let $p>2 n, 0<\kappa<1$ and $\alpha=\frac{p+\kappa-2}{p-1}$. Then there exists a constant $D=$ $D\left(\omega_{K E}\right)>0$ depending on $p, \kappa$ so that

$$
\left\|\phi_{1}-\phi_{t}\right\|_{C^{0}} \leq 100(1-t)\left\|\phi_{t}\right\|_{C^{0}}+1
$$


for all $t \in\left[t_{0}, 1\right]$, where $t_{0}$ (depending on $\phi$ ) is defined by

$$
\left(1-t_{0}\right)^{1-\alpha}\left(1+2\left(1-t_{0}\right)\left\|\phi_{t_{0}}\right\|_{C^{0}}\right)^{\alpha}=\sup _{t \in\left[t_{0}, 1\right]}(1-t)^{1-\alpha}\left(1+2(1-t)\left\|\phi_{t}\right\|_{C^{0}}\right)^{\alpha}=D .
$$

Given Lemma 1, we can complete Step 1. Assume that $p>3-2 \kappa$, so that $\alpha>1 / 2$. Notice that this holds automatically if $n>1$. In the inequality $(2.17)$, we set $t=t_{0}$, where $t_{0}$ is chosen as in Lemma 1. The definition (2.28) of $t_{0}$ implies that

$$
2^{\alpha}\left(1-t_{0}\right)\left\|\phi_{t_{0}}\right\|_{C^{0}}^{\alpha} \leq D\left(\omega_{K E}\right)
$$

Then since $1 / \alpha<2$,

$$
\left(1-t_{0}\right)^{2}\left\|\phi_{t_{0}}\right\|_{C^{0}} \leq D\left(\omega_{K E}\right)^{\frac{1}{\alpha}}
$$

Hence, from the inequality (2.27),

$$
\left(1-t_{0}\right)\left\|\phi_{1}-\phi_{t_{0}}\right\|_{C^{0}} \leq 100\left(1-t_{0}\right)^{2}\left\|\phi_{t_{0}}\right\|_{C^{0}}+1 \leq C_{1},
$$

where $C_{1}$ is another constant depending only on $\omega_{K E}, p$ and $\kappa$. This handles the second term on the right hand side of (2.17). To handle the first term on the right hand side, we begin by noting that we may assume that $1-t_{0}<\delta$ for some fixed small constant $\delta>0$, otherwise the stronger inequality $F_{\omega_{K E}}(\phi) \geq \frac{\delta}{n} J_{\omega_{K E}}(\phi)-\frac{2}{n} C_{1}$ already holds. In particular, we may assume that $\left(1-t_{0}\right)\left\|\phi_{t_{0}}\right\|_{C^{0}} \geq \frac{1}{2}$, for otherwise the definition (2.28) implies that $2^{-\frac{\alpha}{1-\alpha}} D\left(\omega_{K E}\right)^{\frac{1}{1-\alpha}}<1-t_{0}$. When $\left(1-t_{0}\right)\left\|\phi_{t_{0}}\right\|_{C^{0}} \geq \frac{1}{2}$, the definition (2.28) implies

$$
D\left(\omega_{K E}\right) \leq 2^{2 \alpha}\left(1-t_{0}\right)\left\|\phi_{t_{0}}\right\|_{C^{0}}^{\alpha}
$$

giving

$$
1-t_{0} \geq \frac{C_{2}}{\left\|\phi_{t_{0}}\right\|_{C^{0}}^{\alpha}}
$$

The inequality (2.17) implies then

$$
F_{\omega_{K E}}(\phi) \geq C_{2} \frac{J_{\omega_{K E}}(\phi)}{\left\|\phi_{t_{0}}\right\|_{C^{0}}^{\alpha}}-\frac{2}{n} C_{1} .
$$

However, for $\delta \leq \frac{1}{200}$, the inequality (2.27) implies that

$$
\left\|\phi_{t_{0}}\right\|_{C^{0}} \leq 2\left\|\phi_{1}\right\|_{C^{0}}+2
$$

so we obtain

$$
F_{\omega_{K E}}(\phi) \geq C_{2} \frac{J_{\omega_{K E}}(\phi)}{\left(2\left\|\phi_{1}\right\|_{C^{0}}+2\right)^{\alpha}}-\frac{2}{n} C_{1} .
$$


Since

$$
\int_{X} e^{t \phi_{t}} \omega_{\phi_{t}}^{n}=\int_{X} e^{h_{\omega}} \omega^{n}=\int_{X} \omega^{n}=\int_{X} \omega_{\phi_{t}}^{n},
$$

$\phi_{t}$ must change signs. Hence $\left\|\phi_{t}\right\|_{C^{0}} \leq \operatorname{osc}\left(\phi_{t}\right)$. Furthermore osc $\phi_{1}=\operatorname{osc} \phi$ since $\phi_{1}$ and $-\phi$ differ by a constant. Thus we have shown that

$$
F_{\omega_{K E}} \geq C_{2} \frac{J_{\omega_{K E}}(\phi)}{(2 \operatorname{osc} \phi+2)^{\alpha}}-\frac{2}{n} C_{1}
$$

The desired inequality (1.3) with $\gamma=1-\alpha=\frac{1-\kappa}{p-1}$ and all $\phi \in \mathcal{P}\left(X, \omega_{K E}\right)$ satisfying the additional condition (1.8). If $n>1, p$ and $\kappa$ can be taken arbitrarily close to $2 n$ and to 0 respectively and it follows that $\gamma$ can be taken arbitrarily close to $\frac{1}{2 n-1}$. If $n=1$ then we require $p>3-2 \kappa$ giving a $\gamma$ arbitrarily close to $\frac{1}{2}$. This completes Step 1, assuming Lemma 1.

\section{Proof of Lemma 1}

The idea in the proof of Lemma 1 is to smooth out $\phi_{1}-\phi_{t}$ by the Kähler-Ricci flow. More precisely, for each $t$, consider the normalized Kähler-Ricci flow $u_{s, t}$ in time $s$ with initial data $u_{0, t}=0$,

$$
\frac{\partial u_{s, t}}{\partial s}=\log \frac{\left(\omega_{\phi_{t}}+\frac{\sqrt{-1}}{2} \partial \bar{\partial} u_{s, t}\right)^{n}}{\omega_{\phi_{t}}^{n}}+u_{s, t}-h_{\omega_{\phi_{t}}} .
$$

Write $u_{t}$ for $u_{1, t}$, and consider the Kähler form

$$
\omega_{\phi_{t}+u_{t}}=\omega+\frac{\sqrt{-1}}{2} \partial \bar{\partial}\left(\phi_{t}+u_{t}\right)=\omega_{K E}-\frac{\sqrt{-1}}{2} \partial \bar{\partial}\left(\phi_{1}-\phi_{t}-u_{t}\right) .
$$

There exists then a constant $a_{t}$ so that

$$
\log \left(\frac{\omega_{K E}^{n}}{\left(\omega_{K E}-\frac{\sqrt{-1}}{2} \partial \bar{\partial}\left(\phi_{1}-\phi_{t}-u_{t}\right)\right)^{n}}\right)+\phi_{1}-\phi_{t}-u_{t}-a_{t}=h_{\omega_{\phi_{t}+u_{t}}}
$$

which can be seen easily by applying $\sqrt{-1} \partial \bar{\partial}$ to both sides. Set now

$$
\psi_{t}=\phi_{1}-\phi_{t}-u_{t}-a_{t} .
$$

Then the equation (2.21) holds with $\psi=\psi_{t}, h=h_{\omega_{\phi_{t}+u_{t}}}$. The following "smoothing lemma" shows how the bound on $h_{\omega_{\phi_{t}+u_{t}}}$ improves on that of $h_{\omega_{\phi_{t}}}$ :

Lemma 2 Let $u_{t}=u_{1, t}$ be defined as in (3.1). 
(a) We have for all $t \in[0,1]$,

$$
\left\|u_{t}\right\|_{C^{0}} \leq 3\left\|h_{\omega_{\phi_{t}}}\right\|_{C^{0}} .
$$

(b) Let $A>0$ and $t_{1} \in[0,1]$. Assume that

$$
A^{-1} \omega_{K E} \leq \omega_{\phi_{t}+u_{t}} \leq A \omega_{K E},
$$

for all $t \in\left[t_{1}, 1\right]$. Then for any $p>2 n$ and $0<\kappa<1$, there exists a constant $B_{A}$ depending only on $p, A$, and $\omega_{K E}$ so that for $t \in\left[t_{1}, 1\right]$,

$$
\left\|h_{\omega_{\phi_{t}+u_{t}}}\right\|_{C^{0, \kappa}} \leq B_{A}\left(1+\left\|h_{\omega_{\phi_{t}}}\right\|_{C^{0}}\right)^{\alpha}(1-t)^{1-\alpha} .
$$

where $\alpha=\frac{p+\kappa-2}{p-1}$. Here, as before, the norms are with respect to $\omega_{K E}$.

This lemma follows from estimates for the Kähler-Ricci flow and will be proved in the next section. Assuming it for the moment, we complete now the proof of Lemma 1. The next step is to establish the following lemma:

Lemma 3 Fix $p>2 n, 0<\kappa<1$, and choose $D\left(\omega_{K E}\right)=\frac{\epsilon\left(\omega_{K E}\right)}{4\left(B_{2}+1\right)(C+1)(\epsilon+1)}$, where $\epsilon=$ $\epsilon\left(\omega_{K E}\right), C=C\left(\omega_{K E}\right)$ are chosen as in (2.22), and $B_{2}$ is defined as in Lemma 2 with $A=2$. Choose $t_{0}$ as in (2.28). Then for $t \in\left[t_{0}, 1\right]$, we have,

$$
\left\|\psi_{t}\right\|_{C^{2, \kappa}}<\frac{1}{4}
$$

Proof of Lemma 3. We assume the contrary. Since $\psi_{1}=0$, there exists $t_{1} \in\left[t_{0}, 1\right)$ so that

$$
\left\|\psi_{t_{1}}\right\|_{C^{2, \kappa}}=\frac{1}{4} \quad \text { and } \quad\left\|\psi_{t}\right\|_{C^{2, \kappa}}<\frac{1}{4} \quad \text { if } \quad t>t_{1} .
$$

Since the operator norm of any symmetric matrix is always smaller than its HilbertSchmidt norm, we have for $t \in\left[t_{1}, 1\right]$,

$$
-\frac{1}{4} \omega_{K E} \leq \frac{\sqrt{-1}}{2} \partial \bar{\partial} \psi_{t} \leq \frac{1}{4} \omega_{K E},
$$

and thus

$$
\frac{1}{2} \omega_{K E} \leq \omega_{K E}-\frac{\sqrt{-1}}{2} \partial \bar{\partial} \psi_{t}=\omega_{\phi_{t}+u_{t}} \leq 2 \omega_{K E} \quad \text { for } t \in\left[t_{1}, 1\right] .
$$

Thus the inequality (2.20) and Lemma 2 imply that for $t \in\left[t_{1}, 1\right]$,

$$
\begin{aligned}
\left\|h_{\omega_{\phi_{t}+u_{t}}}\right\|_{C^{0, \kappa}} & \leq B_{2}\left(1+\left\|h_{\omega_{\phi_{t}}}\right\|_{C^{0}}\right)^{\alpha}(1-t)^{1-\alpha} \\
& \leq B_{2}\left(1+2(1-t)\left\|\phi_{t}\right\|_{C^{0}}\right)^{\alpha}(1-t)^{1-\alpha} \\
& \leq \frac{\epsilon B_{2}}{4\left(B_{2}+1\right)(C+1)(\epsilon+1)} .
\end{aligned}
$$


This bound is strictly less than $\epsilon$, and thus the bound (2.22) from the implicit function theorem implies for $t \in\left[t_{1}, 1\right]$

$$
\left\|\psi_{t}\right\|_{C^{2, \kappa}} \leq C\left\|h_{\omega_{\phi_{t}+u_{t}}}\right\|_{C^{0, \kappa}} \leq C \frac{\epsilon B_{2}}{4\left(B_{2}+1\right)(C+1)(\epsilon+1)}<\frac{1}{4} .
$$

This contradicts $\left\|\psi_{t_{1}}\right\|_{C^{2, \kappa}}=\frac{1}{4}$, and completes the proof of the lemma. Q.E.D.

Proof of Lemma 1. Still assuming Lemma 2, we can give now the proof of Lemma 1. Since $\phi_{1}-\phi_{t}=\psi_{t}+u_{t}+a_{t}$, and since the bound $\left\|\psi_{t}\right\|_{C^{0}}<1$ is provided by Lemma 3, it will suffice to estimate $\left\|u_{t}\right\|_{C^{0}}$ and $\left|a_{t}\right|$. Using the estimate (2.20) for $h_{\omega_{\phi_{t}}}$, Lemma 2 implies at once $\left\|u_{t}\right\|_{C^{0}} \leq 6(1-t)\left\|\phi_{t}\right\|_{C^{0}}$. Next, the Monge-Ampère equation for $\psi_{t}$ and the normalization of $h_{\omega_{\phi_{t}+u_{t}}}$ give

$$
\int_{X} e^{\psi_{t}} \omega_{K E}^{n}=\int_{X} e^{h_{\omega_{\phi_{t}}+u_{t}}} \omega_{\phi_{t}+u_{t}}^{n}=V
$$

On the other hand, rewriting the Monge-Ampère equation (2.18) in the form $\omega_{K E}^{n}=$ $e^{t \phi_{t}-\phi_{1}} \omega_{\phi_{t}}^{n}$, we also have

$$
\int_{X} e^{\psi_{t}} \omega_{K E}^{n}=\int_{X} e^{\psi_{t}+t \phi_{t}-\phi_{1}} \omega_{\phi_{t}}^{n}=\int_{X} e^{-a_{t}-u_{t}+(t-1) \phi_{t}} \omega_{\phi_{t}}^{n} .
$$

Comparing the two values, we obtain

$$
\left|a_{t}\right| \leq\left\|u_{t}\right\|_{C^{0}}+(1-t)\left\|\phi_{t}\right\|_{C^{0}} \leq 7(1-t)\left\|\phi_{t}\right\|_{C^{0}} .
$$

We can return now to the estimate of $\left\|\phi_{1}-\phi_{t}\right\|_{C^{0}}$,

$$
\left\|\phi_{1}-\phi_{t}\right\|_{C^{0}} \leq\left|a_{t}\right|+\left\|u_{t}\right\|_{C^{0}}+\left\|\psi_{t}\right\|_{C^{0}}
$$

and the desired estimate for $\left\|\phi_{1}-\phi_{t}\right\|_{C^{0}}$ follows immediately. The proof of Lemma 1 is complete. Q.E.D.

\section{Smoothing by the Kähler-Ricci Flow}

We give now the proof of Lemma 2. It is here that the improvement of the exponent in Step 1 over previous results takes place. For convenience, introduce the following notation for the Kähler-Ricci flow,

$$
\frac{\partial u}{\partial s}=\log \frac{\left(\eta_{0}+\frac{\sqrt{-1}}{2} \partial \bar{\partial} u\right)^{n}}{\eta_{0}^{n}}+u-h_{\eta_{0}}, \quad u(0)=0
$$

$\eta_{s}=\eta_{0}+\frac{\sqrt{-1}}{2} \partial \bar{\partial} u$, and $h_{s}=h_{\eta_{s}}$. Then $h_{s}=-\dot{u}+c_{s}=\tilde{h}_{s}+c_{s}$ for some constant $c_{s}$ with $c_{0}=0$. We will use a subscript $s$ to indicate objects that are defined with respect to the metric $\eta_{s}$ (e.g. $\Delta_{s}, \nabla_{s},|\cdot|_{s}$ ). The following lemma is well-known [3], but we include its proof for completeness: 
Lemma 4. We have the following inequalities:

$$
\begin{aligned}
& \|\dot{u}\|_{C^{0}} \leq e^{s}\left\|h_{0}\right\|_{C^{0}} \\
& \sup _{X}\left(\left|h_{s}\right|^{2}+s\left|\nabla h_{s}\right|_{s}^{2}\right) \leq 4 e^{2 s}\left\|h_{0}\right\|_{C^{0}}^{2} \\
& e^{-s} \Delta_{s} h_{s} \geq \Delta_{0} h_{0}
\end{aligned}
$$

Proof of Lemma 4. Differentiating the Kähler-Ricci flow equation gives

$$
\frac{\partial}{\partial s} \dot{u}=\Delta_{s} \dot{u}+\dot{u}
$$

and hence $\|\dot{u}\|_{C^{0}} \leq e^{s}\left\|h_{0}\right\|_{C^{0}}$, giving (a). Similarly, the flows for $\tilde{h}_{s}^{2}$ and $\left|\nabla \tilde{h}_{s}\right|_{s}^{2}$ are,

$$
\begin{aligned}
\frac{\partial}{\partial s} \tilde{h}_{s}^{2} & =\Delta_{s} \tilde{h}_{s}^{2}-2\left|\nabla \tilde{h}_{s}\right|_{s}^{2}+2 \tilde{h}_{s}^{2} \\
\frac{\partial}{\partial s}\left|\nabla \tilde{h}_{s}\right|_{s}^{2} & =\Delta_{s}\left|\nabla \tilde{h}_{s}\right|^{2}-\left|\nabla \bar{\nabla} \tilde{h}_{s}\right|_{s}^{2}-\left|\nabla \tilde{h}_{s}\right|_{s}^{2}+\left|\nabla \tilde{h}_{s}\right|_{s}^{2} .
\end{aligned}
$$

and hence,

$$
\frac{\partial}{\partial s}\left(\tilde{h}_{s}^{2}+s\left|\nabla \tilde{h}_{s}\right|_{s}^{2}\right) \leq \Delta_{s}\left(\tilde{h}_{s}^{2}+s\left|\nabla \tilde{h}_{s}\right|_{s}^{2}\right)+2\left(\tilde{h}_{s}^{2}+s\left|\nabla \tilde{h}_{s}\right|_{s}^{2}\right)
$$

The maximum principle then implies

$$
\sup _{X}\left(e^{-2 s}\left(\tilde{h}_{s}^{2}+s\left|\nabla \tilde{h}_{s}\right|_{s}^{2}\right)\right) \leq\left\|h_{0}\right\|_{C^{0}}^{2}
$$

From the normalization condition for $h_{s}$ and (4.1) we see that

$$
\int_{X} e^{h_{\eta_{0}}+c_{s}-u} \eta_{0}^{n}=V
$$

Part (a) implies $\left|c_{s}\right| \leq e^{s}\left\|h_{0}\right\|_{C^{0}}$ which, when combined with (4.6), yields (b).

Finally, the flow for $\Delta_{s} \dot{u}$ is given by

$$
\frac{\partial}{\partial s}\left(\Delta_{s} \dot{u}\right)=\Delta_{s}^{2} \dot{u}+\Delta_{s} \dot{u}-|\nabla \bar{\nabla} \dot{u}|_{s}^{2} \leq \Delta_{s}^{2} \dot{u}+\Delta_{s} \dot{u}
$$

and (c) follows also from the maximum principle. Q.E.D.

Lemma 5 Assume now that $\eta_{0}=\omega_{\phi_{t}}, h_{0}=h_{\omega_{\phi_{t}}}$. Let $v=h_{1}-\frac{1}{V} \int_{X} h_{1} \eta_{1}^{n}$ and assume that

$$
A^{-1} \omega_{K E} \leq \eta_{1} \leq A \omega_{K E} .
$$

Then for any $p>2 n$, there exists $C>0$, depending only on $\omega_{K E}, A$ and $p$ so that

$$
\|v\|_{C^{0}} \leq C\left\|h_{0}\right\|_{C^{0}}^{\frac{p-2}{p-1}}(1-t)^{\frac{1}{p-1}}
$$


Proof of Lemma 5. Lemma 4 shows that

$$
\|v\|_{C^{0}} \leq 4 e\left\|h_{0}\right\|_{C^{0}}
$$

On the other hand, we also have

$$
\begin{aligned}
\int_{X}|\nabla v|_{1}^{2} \eta_{1}^{n} & =-\int_{X} v\left(\Delta_{1} v\right) \cdot \eta_{1}^{n}=\int_{X}\left(v-\inf _{X} v\right)\left(-\Delta_{1} v\right) \cdot \eta_{1}^{n} \\
& \leq \int_{X}\left(v-\inf _{X} v\right) \sup _{X}\left(-\Delta_{1} v\right) \cdot \eta_{1}^{n} \\
& \leq 2 V\|v\|_{C^{0}} \sup _{X}\left(-\Delta_{1} v\right) .
\end{aligned}
$$

Recall that $h_{0}=h_{\omega_{\phi_{t}}}$ and thus $\operatorname{Ric}\left(\eta_{0}\right)>t \eta_{0}$. This implies that $\Delta_{0} h_{0} \geq-n(1-t)$, and hence, in view of Lemma 4,

$$
-\Delta_{1} h_{1} \leq n e(1-t)
$$

Substituting in the previous inequality gives

$$
\int_{X}|\nabla v|_{1}^{2} \eta_{1}^{n} \leq 2 V n e\|v\|_{C^{0}}(1-t)
$$

Let $p>2 n$. Then for some constants $C_{i}$ depending only on $\omega_{K E}, A$ and $p$,

$$
\begin{aligned}
\|v\|_{C^{0}}^{p} & \leq C_{0}\left(\int_{X}|v|^{p} \eta_{1}^{n}+\int_{X}|\nabla v|_{1}^{p} \eta_{1}^{n}\right) \\
& \leq C_{0}\left(\|v\|_{C^{0}}^{p-2} \int_{X} v^{2} \eta_{1}^{n}+\left(2 e\left\|h_{0}\right\|_{C^{0}}\right)^{p-2} \int_{X}|\nabla v|_{1}^{2} \eta_{1}^{n}\right) \\
& \leq C_{1}\left\|h_{0}\right\|_{C^{0}}^{p-2} \int_{X}|\nabla v|_{1}^{2} \eta_{1}^{n},
\end{aligned}
$$

where we have used the Sobolev inequality, the Poincaré inequality and applied (b) of Lemma 4. Note that the constants in the Sobolev and Poincaré inequalities depend only on $\omega_{K E}$, since the metric $\eta_{1}$ is equivalent to $\omega_{K E}$ in view of (4.9). Together with the inequality (4.14), this gives

$$
\|v\|_{C^{0}}^{p} \leq C_{2}(1-t)\left\|h_{0}\right\|_{C^{0}}^{p-2}\|v\|_{C^{0}}
$$

which is the inequality to be proved. Q.E.D.

Proof of Lemma 2. We can give now the proof of Lemma 2. Recall that $\eta_{0}=\omega_{\phi_{t}}$ and $h_{0}=h_{\omega_{\phi_{t}}}$. From (a) of Lemma 4, it follows that $-e^{s}\left\|h_{0}\right\|_{C^{0}} \leq \dot{u} \leq e^{s}\left\|h_{0}\right\|_{C^{0}}$, and integrating from 0 to 1, we obtain the first statement in Lemma 2. Next, fix $0<\kappa<1$ and let $x, y \in X$. Let $d_{1}(x, y)$ be the distance between $x$ and $y$ with respect to the metric $\eta_{1}$. If $d_{1}(x, y) \geq\left(1+\left\|h_{0}\right\|_{C^{0}}\right)^{-\mu}(1-t)^{\delta}$, for some fixed $0<\delta, \mu \leq 1$ to be chosen later, then Lemma 5 implies

$$
\begin{aligned}
\frac{\left|h_{1}(x)-h_{1}(y)\right|}{d_{1}(x, y)^{\kappa}} & \leq 2\|v\|_{C^{0}}\left(1+\left\|h_{0}\right\|_{C^{0}}\right)^{\mu \kappa}(1-t)^{-\delta \kappa} \\
& \leq C\left(1+\left\|h_{0}\right\|_{C^{0}}\right)^{\frac{p-2}{p-1}+\mu \kappa}(1-t)^{\frac{1}{p-1}-\delta \kappa} .
\end{aligned}
$$


If $d_{1}(x, y)<\left(1+\left\|h_{0}\right\|_{C^{0}}\right)^{-\mu}(1-t)^{\delta}$ instead, then we use the estimate $\left\|\nabla h_{1}\right\|_{C^{0}\left(\eta_{1}\right)} \leq$ $2 e\left\|h_{0}\right\|_{C^{0}}$ from (b) of Lemma 4 to write

$$
\begin{aligned}
\frac{\left|h_{1}(x)-h_{1}(y)\right|}{d_{1}(x, y)^{\kappa}} & \leq d_{1}(x, y)^{1-\kappa}|| \nabla h_{1} \|_{C^{0}\left(\eta_{1}\right)} \\
& \leq C\left(1+\left\|h_{0}\right\|_{C^{0}}\right)^{-\mu(1-\kappa)+1}(1-t)^{\delta(1-\kappa)} .
\end{aligned}
$$

The optimal choice of $\delta, \mu$ is when the exponents of $(1-t)$ and $\left\|h_{0}\right\|_{C^{0}}$ match in the two inequalities, which is $\delta=\mu=\frac{1}{p-1}$. We get then in all cases,

$$
\frac{\left|h_{1}(x)-h_{1}(y)\right|}{d_{1}(x, y)^{\kappa}} \leq C\left(1+\left\|h_{0}\right\|_{C^{0}}\right)^{\alpha}(1-t)^{1-\alpha}
$$

with $\alpha=\frac{p+\kappa-2}{p-1}$. Finally, $\int_{X} e^{h_{1}} \eta_{1}^{n}=\int_{X} \eta_{1}^{n}$ implies that $h_{1}$ changes signs, and thus

$$
\left\|h_{1}\right\|_{C^{0}} \leq \operatorname{osc}\left(h_{1}\right)=\operatorname{osc}(v) \leq 2\|v\|_{C^{0}} \leq C(1-t)^{\frac{1}{p-1}}\left\|h_{0}\right\|_{C^{0}}^{\frac{p-2}{p-1}} .
$$

This is a better bound than the one in (4.19), so we obtain the desired bound for $\left\|h_{1}\right\|_{C^{0, \kappa} \text {. }}$. The proof of Lemma 2, and hence of Step 1, is complete. Q.E.D.

\section{Proofs of the Main Theorems}

In this section we complete Step 2 to prove Theorem 1 and then show how the proof of Theorem 1 can be modified to give a proof of Theorem 2 .

Proof of Theorem 1. We need to prove that for all $\phi \in \mathcal{P}\left(X, \omega_{K E}\right)$,

$$
F_{\omega_{K E}}(\phi) \geq A J_{\omega_{K E}}(\phi)-B
$$

for some $A, B>0$. As before, let $\omega=\omega_{K E}+\frac{\sqrt{-1}}{2} \partial \bar{\partial} \phi$ and let $\phi_{t}$ be the solution of (2.1). We first need the observation of Tian-Zhu [24] that the potential $\phi_{t}-\phi_{1}$ satisfies the condition (1.8),

$$
\operatorname{osc}\left(\phi_{t}-\phi_{1}\right) \leq K\left(J_{\omega_{K E}}\left(\phi_{t}-\phi_{1}\right)+1\right)
$$

for $t \in[1 / 2,1]$ with a constant $K$ depending only on $\omega_{K E}$. We will then prove (5.1) by showing that there exist positive constants $C$ and $c$ depending only on $\omega_{K E}$ such that for some $t$ with $1-t \geq c$,

$$
J_{\omega_{K E}}\left(\phi_{t}-\phi_{1}\right) \leq C .
$$

Recall from section 2 that we have the inequality (2.17),

$$
F_{\omega_{K E}}(\phi) \geq \frac{1}{n}(1-t) J_{\omega_{K E}}(\phi)-\frac{1}{n}(1-t) \operatorname{osc}\left(\phi_{t}-\phi_{1}\right),
$$


for any $t \in[0,1]$. Then this together with (5.2) and (5.3) will give (5.1).

To prove (5.2) observe that $\operatorname{Ric}\left(\omega_{\phi_{t}}\right) \geq \frac{1}{2} \omega_{\phi_{t}}$ when $t$ is in the interval $[1 / 2,1]$. It follows (see [18], for example) that the Green's function for $\omega_{\phi_{t}}$ is uniformly bounded from below. Since $\omega_{K E}+\frac{\sqrt{-1}}{2} \partial \bar{\partial}\left(\phi_{t}-\phi_{1}\right)=\omega_{\phi_{t}}>0$ and $\omega_{\phi_{t}}+\frac{\sqrt{-1}}{2} \partial \bar{\partial}\left(\phi_{1}-\phi_{t}\right)=\omega_{K E}>0$ we have

$$
-\Delta_{\omega_{K E}}\left(\phi_{t}-\phi_{1}\right)<n \text { and }-\Delta_{t}\left(\phi_{t}-\phi_{1}\right)>-n \text {. }
$$

Then using the Green's functions for $\omega_{K E}$ and $\omega_{\phi_{t}}$ there exists $C_{0}=C_{0}\left(\omega_{K E}\right)$ such that for all $x, y$ in $X$,

$$
\begin{aligned}
& \left(\phi_{t}-\phi_{1}\right)(x) \leq \frac{1}{V} \int_{X}\left(\phi_{t}-\phi_{1}\right) \omega_{K E}^{n}+C_{0} \\
& \left(\phi_{t}-\phi_{1}\right)(y) \geq \frac{1}{V} \int_{X}\left(\phi_{t}-\phi_{1}\right) \omega_{\phi_{t}}^{n}-C_{0} .
\end{aligned}
$$

Hence, using (2.2) and (2.3),

$$
\begin{aligned}
\operatorname{osc}\left(\phi_{t}-\phi_{1}\right) & \leq \frac{1}{V} \int_{X}\left(\phi_{t}-\phi_{1}\right)\left(\omega_{K E}^{n}-\omega_{\phi_{t}}^{n}\right)+2 C_{0} \\
& =I_{\omega_{K E}}\left(\phi_{t}-\phi_{1}\right)+2 C_{0} \leq K\left(J_{\omega_{K E}}\left(\phi_{t}-\phi_{1}\right)+1\right),
\end{aligned}
$$

as required.

We will now give a proof of (5.3). Making use of the cocycle condition for $F$, the equations (2.1), (2.7), (2.8) and the concavity of the log function, we have for $t \in(0,1]$,

$$
\begin{aligned}
F_{\omega_{K E}}\left(\phi_{t}-\phi_{1}\right)= & F_{\omega}\left(\phi_{t}\right)-F_{\omega}\left(\phi_{1}\right) \\
= & -\frac{1}{t} \int_{0}^{t}\left(I_{\omega}-J_{\omega}\right)\left(\phi_{s}\right) d s+\int_{0}^{1}\left(I_{\omega}-J_{\omega}\right)\left(\phi_{s}\right) d s-\log \left(\frac{1}{V} \int_{X} e^{(t-1) \phi_{t}} \omega_{\phi_{t}}^{n}\right) \\
\leq & -\frac{1}{t} \int_{0}^{t}\left(I_{\omega}-J_{\omega}\right)\left(\phi_{s}\right) d s+\int_{0}^{1}\left(I_{\omega}-J_{\omega}\right)\left(\phi_{s}\right) d s+\frac{(1-t)}{V} \int_{X} \phi_{t} \omega_{\phi_{t}}^{n} \\
= & -\frac{1}{t} \int_{0}^{t}\left(I_{\omega}-J_{\omega}\right)\left(\phi_{s}\right) d s+\int_{0}^{1}\left(I_{\omega}-J_{\omega}\right)\left(\phi_{s}\right) d s-(1-t)\left(I_{\omega}-J_{\omega}\right)\left(\phi_{t}\right) \\
& +\frac{(1-t)}{t} \int_{0}^{t}\left(I_{\omega}-J_{\omega}\right)\left(\phi_{s}\right) d s \\
= & \int_{t}^{1}\left(I_{\omega}-J_{\omega}\right)\left(\phi_{s}\right) d s-(1-t)\left(I_{\omega}-J_{\omega}\right)\left(\phi_{t}\right) \\
\leq & (1-t)\left(\left(I_{\omega}-J_{\omega}\right)\left(\phi_{1}\right)-\left(I_{\omega}-J_{\omega}\right)\left(\phi_{t}\right)\right) \\
\leq & n(1-t) \operatorname{osc}\left(\phi_{t}-\phi_{1}\right),
\end{aligned}
$$

where for the last line we have used inequality (2.14) and in the previous line, we have used the fact that $\left(I_{\omega}-J_{\omega}\right)\left(\phi_{s}\right)$ is nondecreasing in $s$. From (5.2) we see that for $t \in[1 / 2,1]$,

$$
F_{\omega_{K E}}\left(\phi_{t}-\phi_{1}\right) \leq n(1-t) K\left(J_{\omega_{K E}}\left(\phi_{t}-\phi_{1}\right)+1\right) .
$$


But from Step 1 we have

$$
A_{\gamma} J_{\omega_{K E}}\left(\phi_{t}-\phi_{1}\right)^{\gamma}-B_{\gamma} \leq F_{\omega_{K E}}\left(\phi_{t}-\phi_{1}\right)
$$

and combining this with the preceding inequality gives

$$
J_{\omega_{K E}}\left(\phi_{t}-\phi_{1}\right)^{\gamma}\left(A_{\gamma}-n K(1-t) J_{\omega_{K E}}\left(\phi_{t}-\phi_{1}\right)^{1-\gamma}\right) \leq n(1-t) K+B_{\gamma} .
$$

We can suppose that there exists a $t^{\prime} \in[1 / 2,1]$ with

$$
n K\left(1-t^{\prime}\right) J_{\omega_{K E}}\left(\phi_{t^{\prime}}-\phi_{1}\right)^{1-\gamma}=A_{\gamma} / 2 \text {. }
$$

For, if not then we must have

$$
n K(1-t) J_{\omega_{K E}}\left(\phi_{t}-\phi_{1}\right)^{1-\gamma}<A_{\gamma} / 2 \text {, }
$$

for all $t \in[1 / 2,1]$ and hence for $t=1 / 2$. It would follow that $J_{\omega_{K E}}\left(\phi_{1 / 2}-\phi_{1}\right) \leq C$, and we would be done. Otherwise, from (5.12) we see that $J_{\omega_{K E}}\left(\phi_{t^{\prime}}-\phi_{1}\right) \leq C$ and from (5.13) that $1-t^{\prime} \geq c>0$ and we are finished. Q.E.D.

Proof of Theorem 2. The assumption of no non-trivial holomorphic vector fields is used in the proof of Theorem 1 for two applications of the implicit function theorem. The first application is to obtain a solution $\phi_{t}$ of (2.1) and the second is to obtain the estimate of Lemma 3. In both cases, the key fact is that the operator $f \mapsto \Delta_{\omega_{K E}} f+f$ is an invertible operator from $C^{2, \kappa}(X)$ to $C^{\kappa}(X)$. The kernel $\Lambda$ of this operator will be nontrivial if there are holomorphic vector fields. Let $G$ be as in Theorem 2 , and let $\lambda_{1}, \cdots, \lambda_{M}$ be a basis of $\Lambda$, and define for each $g \in G$ the matrix $a_{i j}(g)$ by $\rho(g) \lambda_{i}=a_{i j}(g) \lambda_{j}$, where $\rho$ is the action of $G$ on $\Lambda$. Then if $f$ is $G$-invariant, we have

$$
V_{i} \equiv \int_{X} f \lambda_{i} \omega_{K E}^{n}=a_{i j}(g) \int_{X} f \lambda_{j} \omega_{K E}^{n}=a_{i j}(g) V_{j},
$$

which means that the vector $V_{i}$ defined by the left hand side is fixed under $G$. Since $G$ has by assumption no non-zero fixed vector, the vector $V_{i}$ must be 0 , which shows that $f$ is orthogonal to $\Lambda$. Hence replacing $C^{2, \kappa}(X)$ and $C^{\kappa}(X)$ with their $G$-invariant counterparts, we see that the operator is still invertible in this case as long as the objects involved are $G$-invariant. But $\omega$ and $h_{\omega}$ are $G$-invariant since $\omega_{K E}$ and $\phi$ are, and it follows that the family $\phi_{t}$ is $G$-invariant. Since the Ricci flow preserves the $G$-invariance, $u_{t}$ and $\psi_{t}$ are $G$-invariant. The proof of Step 1 then goes through as before. Step 2 follows as above once we observe that $\phi_{t}-\phi_{1}$ is $G$-invariant. Q.E.D.

Acknowledgements: We would like to thank the referee for pointing out some inaccuracies in the original version of this manuscript.

\section{References}

[1] Aubin, T. Équations du type Monge-Ampère sur les variétés kählériennes compactes, Bull. Sci. Math. (2) 102 (1978), no. 1, 63-95 
[2] Aubin, T. Meilleures constantes dans le théorème d'inclusion de Sobolev et un théorème de Fredholm non linéaire pour la transformation conforme de la courbure scalaire, J. Funct. Anal. 32 (1979), no. 2, 148-174

[3] Bando, S. The K-energy map, almost Einstein Kähler metrics and an inequality of the Miyaoka-Yau type, Tohuku Math. Journ. 39 (1987), 231-235

[4] Bando, S. and Mabuchi, T. Uniqueness of Einstein Kähler metrics modulo connected group actions, Adv. Stud. in Pure Math. 10 (1987), 11-40

[5] Beckner, W. Sharp Sobolev inequalities on the sphere and the Moser-Trudinger inequality, Ann. of Math. (2) 138 (1993), no. 1, 213-242

[6] Cao, H.-D., G. Tian, and X. Zhu, Kähler-Ricci solitons on compact complex manifolds with $c_{1}(M)>0$, Geom. Funct. Anal. 15 (2005), 679-719

[7] Chang, S.Y.A. and Yang, P., Prescribing Gaussian curvature on $S^{2}$, Acta Math. 159 (1987), no. $3-4,215-259$.

[8] Chen, X.X. and Tian, G. Ricci flow on Kähler-Einstein surfaces, Invent. Math. 147 (2002), $487-544$

[9] Ding, W. Remarks on the existence problem of positive Kähler-Einstein metrics, Math. Ann. 282 (1988), no. 3, 463-471

[10] Donaldson, S.K. Anti self-dual Yang-Mills connections over complex algebraic surfaces and stable vector bundles, Proc. London Math. Soc. (3) 50 (1985), no. 1, 1-26

[11] Donaldson, S.K. Infinite determinants, stable bundles and curvature, Duke Math J. 54 (1987), no. 1, 231-247

[12] Lichnerowicz, A. Sur les transformations analytiques des variétés kählériennes compactes, C. R. Acad. Sci. Paris 244 (1957), 3011-3013

[13] Mabuchi, T. K-energy maps integrating Futaki invariants, Tôhoku Math. Journ. 38 (1986), $575-593$

[14] Matsushima, Y. Sur la structure du groupe d'homéomorphismes analytiques d'une certaine variété kählérienne, Nagoya Math. J. 11 (1957), 145-150

[15] Moser, J. A sharp form of an inequality by N. Trudinger, Indiana Univ. Math. J. 20 (1971), 1077-1091

[16] Onofri, E. On the positivity of the effective action in a theory of random surfaces, Comm. Math. Phys. 86 (1982), no. 3, 321-326

[17] Sano, Y. The best constant of the Moser-Trudinger inequality on $S^{2}$, Trans. Amer. Math. Soc. 356 (2004), no. 9, 3477-3482 
[18] Siu, Y.-T. Lectures on Hermitian-Einstein metrics for stable bundles and Kähler-Einstein metrics. DMV Seminar, 8. Birkhäuser Verlag, Basel, 1987

[19] Song, J. The $\alpha$-invariant on certain surfaces with symmetry groups, Trans. Amer. Math. Soc. 357 (2005), no. 1, 45-57

[20] Song, J. and Weinkove, B. Energy functionals and canonical Kähler metrics, to appear in Duke Math. J.

[21] Tian, G. On Kähler-Einstein metrics on certain Kähler manifolds with $c_{1}(M)>0$, Invent. Math. 89 (1987), 225-246

[22] Tian, G. Kähler-Einstein metrics with positive scalar curvature, Invent. Math. 137 (1997), $1-37$

[23] Tian, G. and Yau, S.-T. Kähler-Einstein metrics on complex surfaces with $c_{1}>0$, Comm. Math. Phys. 112 (1987), no. 1, 175-203

[24] Tian, G. and Zhu, X. A nonlinear inequality of Moser-Trudinger type, Calc. Var. 10 (2000), $349-354$

[25] Trudinger, N. S. On imbeddings into Orlicz spaces and some applications, J. Math. Mech. 17 (1967), 473-483

[26] Uhlenbeck, K. and Yau, S.-T. On the existence of Hermitian-Yang-Mills connections on stable vector bundles Comm. Pure Appl. math. 39 (1986), no. S, suppl., S257-S293

[27] Yau, S.-T. On the Ricci curvature of a compact Kähler manifold and the complex MongeAmpère equation, I, Comm. Pure Appl. Math. 31 (1978), no. 3, 339-411

[28] Yau, S.-T. Open problems in geometry, Proc. Symposia Pure Math. 54 (1993), 1-28 (problem $65)$

[29] Zhou, B. and Zhu X. Relative K-stability and modified K-energy on toric manifolds, preprint, math.DG/0603237 